\title{
Core of the saliva microbiome: an analysis of the MG-RAST data
}

\author{
Simone G. Oliveira ${ }^{1,2+}$, Rafaela R. Nishiyama' ${ }^{1}$ Claudio A. C. Trigo ${ }^{1}$, Ana Luiza Mattos-Guaraldi³ \\ Alberto M. R. Dávila ${ }^{4}$, Rodrigo Jardim ${ }^{4^{*}+}$ and Flavio H. B. Aguiar ${ }^{1}$
}

\begin{abstract}
Background: Oral microbiota is considered as the second most complex in the human body and its dysbiosis can be responsible for oral diseases. Interactions between the microorganism communities and the host allow establishing the microbiological proles. Identifying the core microbiome is essential to predicting diseases and changes in environmental behavior from microorganisms.
\end{abstract}

Methods: Projects containing the term "SALIVA", deposited between 2014 and 2019 were recovered on the MG-RAST portal. Quality (Failed), taxonomic prediction (Unknown and Predicted), species richness (Rarefaction), and species diversity (Alpha) were analyzed according to sequencing approaches (Amplicon sequencing and Shotgun metagenomics). All data were checked for normality and homoscedasticity. Metagenomic projects were compared using the Mann-Whitney $U$ test and Spearman's correlation. Microbiome cores were inferred by Principal Component Analysis. For all statistical tests, $p<0.05$ was used.

Results: The study was performed with 3 projects, involving 245 Amplicon and 164 Shotgun metagenome datasets. All comparisons of variables, according to the type of sequencing, showed significant differences, except for the Predicted. In Shotgun metagenomics datasets the highest correlation was between Rarefaction and Failed $(r=-0.78$ ) and the lowest between Alpha and Unknown $(r=-0.12)$. In Amplicon sequencing datasets, the variables Rarefaction and Unknown $(r=0.63)$ had the highest correlation and the lowest was between Alpha and Predicted $(r=-0.03)$. Shotgun metagenomics datasets showed a greater number of genera than Amplicon. Propionibacterium, LactobacilIus, and Prevotella were the most representative genera in Amplicon sequencing. In Shotgun metagenomics, the most representative genera were Escherichia, Chitinophaga, and Acinetobacter.

Conclusions: Core of the salivary microbiome and genera diversity are dependent on the sequencing approaches. Available data suggest that Shotgun metagenomics and Amplicon sequencing have similar sensitivities to detect the taxonomic level investigated, although Shotgun metagenomics allows a deeper analysis of the microorganism diversity. Microbiome studies must consider characteristics and limitations of the sequencing approaches. Were identified 20 genera in the core of saliva microbiome, regardless of the health condition of the host. Some bacteria of the core need further study to better understand their role in the oral cavity.

Keywords: Microbiome core, Saliva, MG-RAST, Amplicon sequencing, Shotgun metagenomics, eHOMD

\section{*Correspondence: rodrigo.jardim@fiocruz.br}

${ }^{\dagger}$ Simone G. Oliveira and Rodrigo Jardim have contributed equally to this work

${ }^{4}$ Computational and Systems Biology Laboratory, Oswaldo Cruz Institute, Oswaldo Cruz Foundation, Av. Brasil, 4365, Rio de Janeiro, Brazil Full list of author information is available at the end of the article

\section{Background}

Metagenomic is a technique for accessing non-cultivable microorganisms DNA from environmental samples [1]. Since the development of New Generation Sequencing (NGS), this technique has been widely used in a number 
of scientific studies [2-4]. However, one of the challenges in metagenomics is to work with a large volume of data generated by sequencing and analysis. In bioinformatics, sequencing data must be deposited in a public database for wide access to be published in a scientific article. Therefore, since 2008 several specialized databases have allowed the deposit of raw and analyzed data from metagenomics projects $[5,6]$. One of the pioneers in storage platforms for metagenomic data analysis is the public access portal MG-RAST [6].

The MG-RAST portal has deposited projects generated from different metagenomics approaches: Amplicon sequencing, Shotgun metagenomics and Metatranscriptomic. Amplicon sequencing (or metabarcoding) is done using the products of the polymerase chain reaction (PCR) that amplify the marker genes, such as 16S rRNA, $23 \mathrm{~S}$ rRNA and $18 \mathrm{~S}$ rRNA. Shotgun metagenomics has been used for total DNA sequencing from environmental samples while Metatranscriptomic has been used for sequencing all RNA extracted from investigated samples.

The oral microbiota refers to the collection of microorganisms that inhabit the oral cavity in different locations, such as the tongue, saliva and teeth [7]. Saliva has been shown to be a biological material capable of reflecting the dynamics of health conditions and metabolic, immunological or infectious diseases, reflecting dysbiosis of local and systemic origin, as observed in dental caries, periodontal diseases, diabetes, rheumatoid arthritis [8], cancer and, more recently, SARS-COV-2 [9, 10]. Obtaining salivary samples is simple, easy to perform, non-invasive, does not cause discomfort to the patient, is inexpensive, and representative of the oral environment. Saliva, like other biological fluids, contains DNA, RNA, proteins, and metabolic products, which are components of the host and its microbiota and their interactions. Despite the ease of its collection, knowledge of the behavior of the salivary microbiota and other constituents is still a challenge. Studies using sophisticated analysis such as the NGS, allow the investigation of differences in the bacterial profile in patients with oral diseases, such as caries and periodontitis, compared to healthy individuals [11].

The oral human microbiome, considered as the second most complex, is composed of more than 700 species [12] of which $54 \%$ are cultivable, $14 \%$ cultivable but unidentifiable, in addition to $32 \%$ of microorganisms unable to be cultured and identified [13].

Recent studies have proven the existence of an intrinsic relationship between the environment conditions and microbiome profiles $[3,13,14]$. Oral microbiota dysbiosis can be responsible for oral diseases, such as caries, plaque and periodontics [15-17]. Host-microbiological interactions allow establishing differences in microbiome profiles due to physiological and pathological conditions
[18]. During the last decades, the development of methodologies and analyses for the identification and characterization of microbiomes has made it possible to predict diseases associated with changes in the environment and their reflexes in the microbiota, particularly those that share the same niche [19].

Description of microorganisms that share the same niche is called a core [19]. Identifying the core microbiome is essential to define how "healthy" this environment is [19]. The literature points out that the $10 \%$ prevalent microorganisms in the core must be considered dominant. On the other hand, the $65 \%$ less predominant should be considered rare [20].

Therefore, the relationship of this core with the environment, predicting diseases, and changes in environmental behavior from microorganisms not belonging to the core need further investigation [19].

\section{Main text \\ Methods}

This study aimed to investigate the core of the oral microbiome in saliva samples, regardless of host conditions by using the MG-RAST portal database.

Identifiers of metagenomes deposited between 2014 and 2019 , containing as keyword the term "saliva" in the Material variable (material='SALIVA') were selected for the study. A python in-house script was developed to extract, transform and load metadata from selected metagenomes to be filtered and analyzed. In order to assess the differences between sequencing approaches, only metagenomic projects that contained Amplicon sequencing and Shotgun metagenomics approaches were used in this study. In addition, only projects that had more than 3 metagenomes in each of the approaches were used due to statistical inferences.

Metagenomes were analyzed according to quality (Failed) and taxonomic prediction (Unknown and Predicted). In addition, metagenomes were analyzed according to species richness (Rarefaction) and species diversity (Alpha) (Table 1). Taxonomic data were recovered from the projects selected for the study of the core of the microbiome. Genus level was chosen for the analysis because Amplicon sequencing approach is more consistent for this taxonomic level [21].

All data were checked for normality and homoscedasticity by Shapiro-Wilk test. Amplicon sequencing and Shotgun metagenomics data were compared using the Mann-Whitney U test. Associations among Failed, Unknown, Predicted, Alpha, and Rarefaction were performed by Spearman's correlation. Microbiome cores were obtained and analyzed by Principal Component Analysis (PCA), to evaluate the most representative 
Table 1 Variables recovered in MG-RAST

\begin{tabular}{lll}
\hline Variable & Description & MG-RAST name \\
\hline Failed & Low quality sequences & QC failed \\
Unknown & $\begin{array}{c}\text { Unrecognized sequence in public } \\
\text { databases }\end{array}$ & QC unknown \\
Predicted & Sequence with inferred taxonomy & QC predicted \\
Alpha & Alpha diversity by Shannon index & ALPHA \\
Rarefaction & Max value of rarefaction curve & RAREFACTION \\
Type & Sequencing approaches & Sequence type \\
Taxonomy & Taxonomy at genus level & Taxonomy
\end{tabular}

The variables Failed, Unknown, Predicted, Alpha, and Rarefaction were used in the descriptive and correlation analyses. Type represents the sequencing approaches (Amplicon sequencing and Shotgun metagenomics). The Taxonomy variable contains the genera found in the samples that were used in the Principal Components Analysis

organisms of Amplicon sequencing and Shotgun metagenomics.

To allow a comparison between the results obtained in this study and the current literature, representative data of the oral microbiome from the expanded Human Oral Microbiome Database (eHOMD) [22] were used. eHOMD data were retrieved using the Taxon Table available at http://www.homd.org/?name=HOMD. After setting the Body Site filter field by checking only the Oral option, the text file was downloaded. Only the information in the Genus column was considered. Then the redundancies were removed and the remaining genera were considered the microbiome core of eHOMD. Microbiome cores obtained in the Amplicon sequencing and Shotgun metagenomics were compared with the microbiome core of the eHOMD by Venn diagram.

Statistical analysis was performed using the software $\mathrm{R}$ v3.6.1 [23]. The libraries dplyr, ggplot2, reshape2, and data.table were used in the descriptive analysis and correlation study. The factoextra library was used in the PCA. The value of $p<0.05$ was used in all tests.

Python and R scripts are available on GitHub (https:// github.com/rodrigojardim/mgrast-search).

In order to obtain more information about the selected projects, a search was carried out on PUBMED using the information of the principal investigator and the description of the project, both available on MG-RAST.
Table 2 Number of metagenomes by project and sequencing approaches

\begin{tabular}{lll}
\hline Project & Approaches & $\begin{array}{l}\text { Number of } \\
\text { metagenomes }\end{array}$ \\
\hline mgp3474 & Amplicon & 95 \\
mgp4843 & Amplicon & 97 \\
mgp7236 & Amplicon & 53 \\
mgp3474 & Shotgun & 8 \\
mgp4843 & Shotgun & 73 \\
mgp7236 & Shotgun & 83 \\
\hline
\end{tabular}

\section{Results}

The survey in MG-RAST identified 621 metagenomes. Python script recovered 476 metagenomes, distributed in 12 sequencing projects, of which 332 metagenomes were Amplicon sequencing, 142 Shotgun metagenomics, and 2 Metatranscriptomes. The remaining 145 metagenomes had no metadata available and were discarded, as well as the recovered metatranscriptomes.

Projects that contained metagenomes from both sequencing approaches were selected. In addition, 1 project that had only 1 metagenome for each approach was discarded. In this way, 3 projects with 245 Amplicon sequencing metagenomes and 164 Shotgun metagenomics metagenomes were used in this study (Table 2).

Figure 1 shows the results of the descriptive analysis of the numerical variables. For the Amplicon sequencing datasets there was no sequence with quality problems. All variables of both approaches had a non-Gaussian distribution, with the exception of the Alpha in Shotgun metagenomics. All comparisons of variables, according to the sequencing approaches, showed significant differences $(p<0.05)$, except for the Predicted $(p=0.4307)$.

Correlation study of the five variables showed divergences between sequencing approaches (Fig. 2). Most of the correlations of the variables in Amplicon sequencing datasets were positive, in contrast to what was observed in Shotgun metagenomics. In Shotgun datasets, the highest correlation was between Rarefaction and Failed $(r=-0.78)$ and the lowest between Alpha and Unknown $(r=-0.12)$. In Amplicon datasets, Rarefaction and Unknown $(r=0.63)$ had the highest correlation and the lowest was between Alpha and Predicted $(r=-0.03)$.

\footnotetext{
(See figure on next page.)

Fig. 1 Descriptive analysis. Boxplot shows the data distribution of Failed, Unknown, Predicted, Alpha and Rarefaction variables, comparing (a) Amplicon sequencing and (b) Shotgun metagenomics approaches. Normality analysis was performed using the Shapiro Wilk test and the MannWhitney $\mathrm{U}$ test evaluated the differences of the variables between the sequencing approaches. All variables showed a significant difference $(p<0.05)$ between Amplicon sequencing and Shotgun metagenomics, except Predicted. Note that Failed did not present any data in the Amplicon sequencing
} 


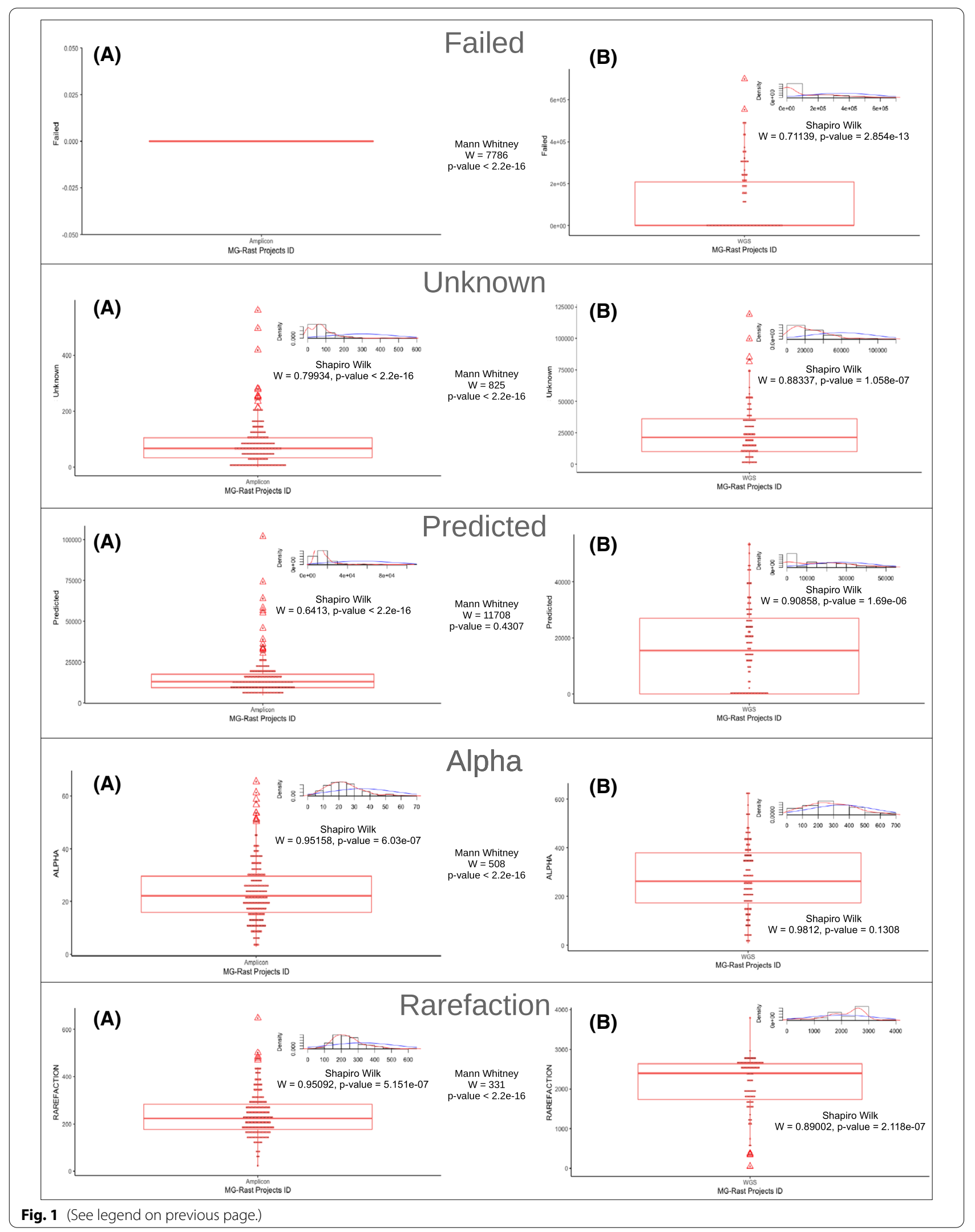


(A)

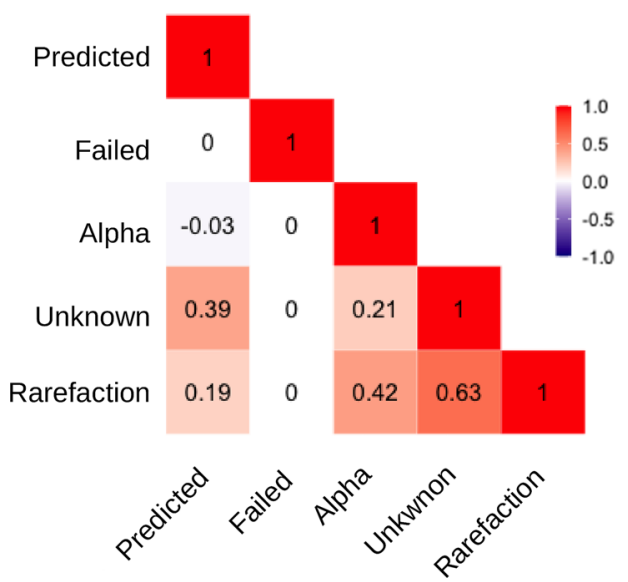

(B)

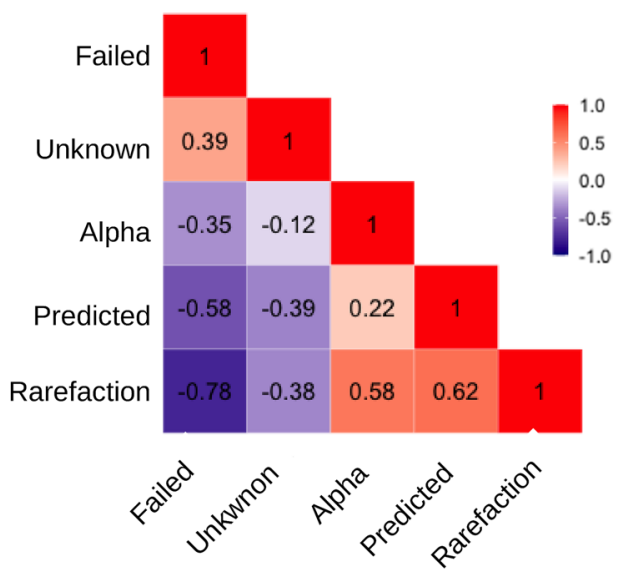

Fig. 2 Correlation analysis. The Spearman's test evaluated the associations between Failed, Unknown, Predicted, Alpha, and Rarefaction variables of a Amplicon sequencing and $\mathbf{b}$ Shotgun metagenomics. All associations were positive in Amplicon sequencing. In the Shotgun metagenomics, Failed and Unknown were negatively associated with Predicted, Alpha, and Rarefaction

In the PCA, microbiome cores with different genera were found between Amplicon sequencing and Shotgun metagenomics. Number of genera obtained by the Shotgun dataset was greater than that observed in the Amplicon dataset. The top 10 genera showed that only Prevotella and Streptococcus are representative in cores of both approaches. Propionibacterium, Lactobacillus and Prevotella were the most representative genera in Amplicon sequencing. On the other hand, Escherichia, Chitinophaga, and Acinetobacter (Figs. 3 and 4) were the most representative genera in Shotgun metagenomics. The genera present in the microbiome core of both Shotgun and Amplicon are listed in Additional file 1: S1.

By comparing Shotgun metagenomics, Amplicon sequencing and eHOMD datasets a common or shared microbiome core containing 20 genera was found (Table 3). eHOMD and Shotgun metagenomics datasets share a microbiome core composed of 39 genera, and eHOMD and Amplicon sequencing share a core with only 9 genera. Shotgun and Amplicon datasets share a core containing 7 genera (Fig. 5). The complete genera names sharing the different microbiome cores is in Additional file 2: S2.

Search in PUBMED identified 12 articles published between 2011 and 2015, with the PMIDs: 25994215, 25861745, 25020228, 24981669, 24903519, 24846382, 24646696, 23598790, 22583485, 2254158393, 21149389, and 205478343.

\section{Discussion}

Bacteria are highly prevalent microorganisms in the microbiota and play an important role in oral homeostasis [41]. The abundance of some bacteria may indicate dysbiosis of the oral microbiome [25, 35]. Identification of the core is inferred from the study of different microbiomes and allows to reveal the conditions of the hosts according to the presence/absence or predominance of some species over others. However, there is a set of bacteria that, regardless of the condition of the host, can be part of the core of all these microbiomes. Similar to the concept of housekeeping genes, which conceptualize the genes essential to the life of an organism, a set of bacteria from the oral microbiome, regardless of the health status of the host, can be inferred as that essential to the symbiosis between the microorganisms of the oral cavity.

In this study, 20 genera of bacteria were found in more than 450 metagenomes (Fig. 5) deposited in public databases and which, regardless of the health condition of the host, are present in the core of the oral microbiome.

In both Amplicon sequencing and Shotgun metagenomics cores, genera of bacteria already associated with caries and periodontal diseases were found, such as Streptococcus, Lactobacillus and Prevotella [10, 42].

The saliva of individuals with high caries experience is associated with a high salivary abundance of Streptococcus and countless species of Lactobacillus in addition to other bacteria capable of degrading sugars and forming extracellular polysaccharides [18]. Samples from healthy 

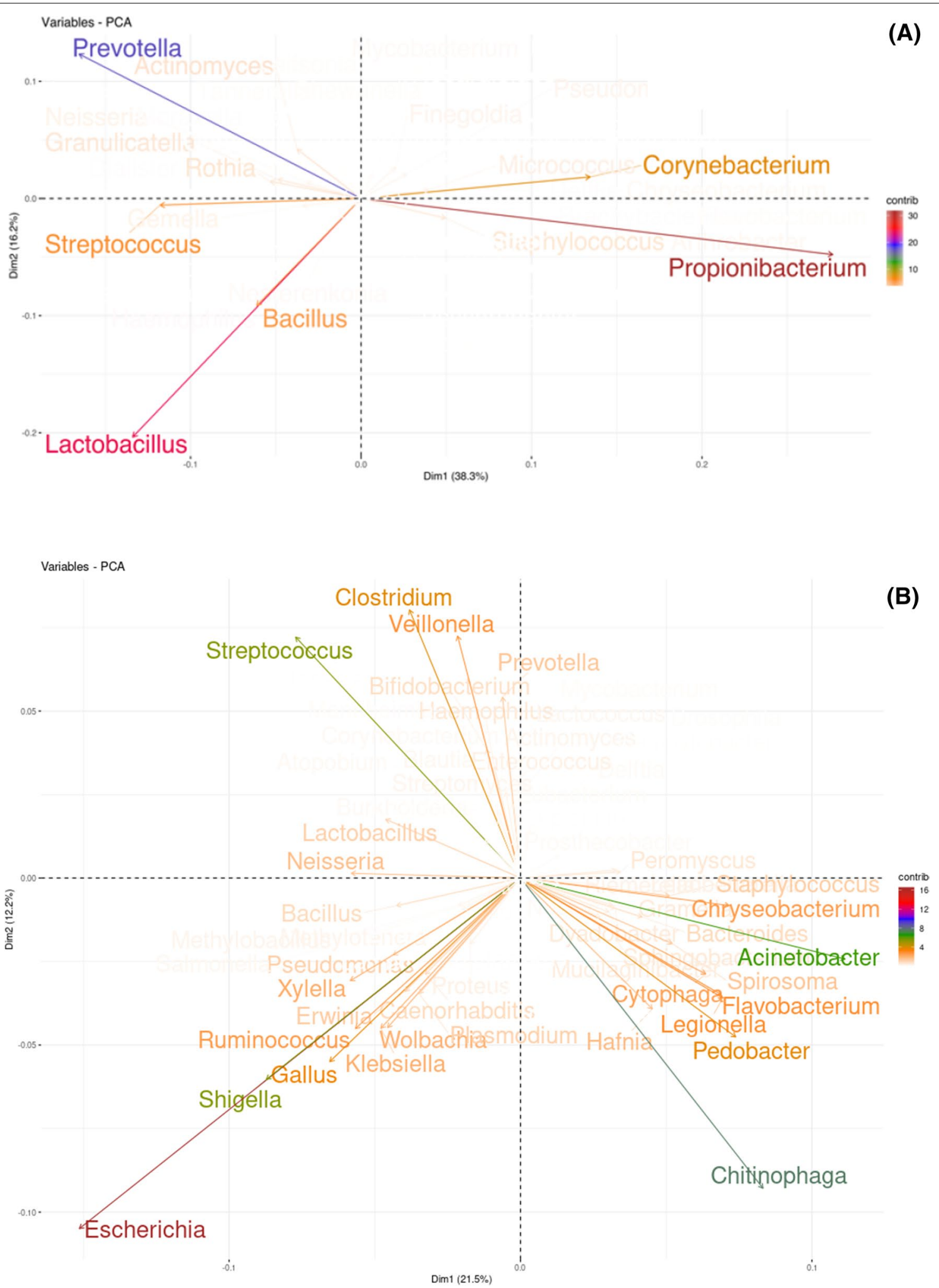

Fig. 3 Principal Components Analysis. Bi-plot showing the representativeness of the genera present in the core of both approaches: a Amplicon sequencing and $\mathbf{b}$ Shotgun metagenomics. Graphs show the dimensions with greatest variance. 


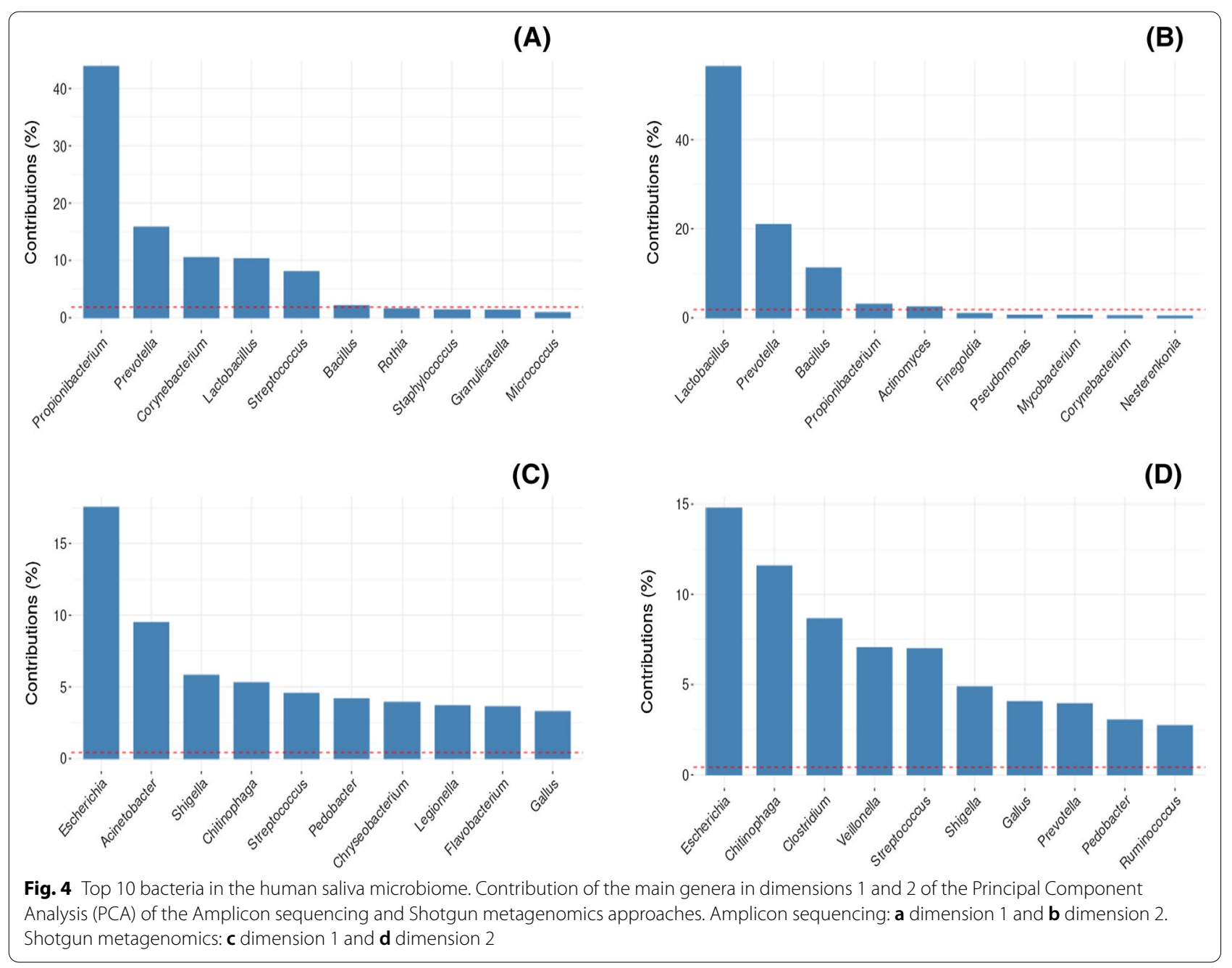

individuals with low caries experience were associated with a greater abundance of the genera Neisseria, Haemophilus, and Fusobacterium, of which most species of this genera only ferment sugar slightly [43]. According to Tanner et al. [18], the composition of saliva in the oral cavity is one of the main risk factors associated with caries. Biofilm dysbiosis results in an increase in acidogenic and aciduric species, capable of modulating the core components in the biofilm. While in cases of gingivitis, the increase in the amount of plaque around the gingival margin induces the inflammatory response in the host, leading to increased levels of anaerobic bacteria, including Gram-negative proteolytic species, especially those belonging to the Prevotella, Porphyromonas, Tannerella, Fusobacterium and Treponema genera [44].

Identification of Corynebacterium, Escherichia, Pseudomonas and Shigella suggests that genera with pathogenic potential may also be part of the core of the oral microbiome obtained by salivary samples. Chitinophaga was a recently described taxon and was observed only in
Shotgun metagenomics. This genus was highly representative, as well as Escherichia, Acinetobacter, Streptococcus, and Shigella (Fig. 3). The pathogenic potential of the genus Chitinophaga has already been reported $[45,46]$. However, its role in the oral microbiome is still unknown.

Amplicon sequencing metadata analysis showed inconsistent behavior (Fig. 2). Only Rarefaction and Unknown had correlation greater than $0.50(r=0.63)$. The expected behavior was of inverse correlation. The greatest number of non-inferred sequences determines the smallest number of potentially discovered organisms.

On the other hand, Shotgun metagenomics presented results as expected, which can be exemplified by inverse correlation between Failure and Rarefaction. The largest number of sequences with quality failure determines the smallest number of sequences to be inferred, which affects the rarefaction curve. This was exactly the behavior observed in Shotgun metagenomics for these variables $(r=-0.78)$. 
Table 3 Bacteria genera of human saliva microbiome core and their relationship with oral diseases

\begin{tabular}{lll}
\hline Gram staining & Genus & Relation with oral disease \\
\hline Gram-negative & Acinetobacter & Oral squamous cell carcinoma [24] \\
& Delftia & Geographic tongue [25] \\
& Dialister & Sjøgren's syndrome [26] \\
& Enterobacter & Denture stomatitis [27] \\
& Haemophilus & Squamous cell carcinoma [28] \\
& Moraxella & Peri-implantitis [29] \\
& Neisseria & Healthy periodontal conditions [30] \\
& Prevotella & Periodontal disease [30] \\
& Pseudomonas & Oral cancer [31] \\
& Ralstonia & Periodontitis [32] \\
& Sphingomonas & Recurrent aphthous ulcer [33] \\
& Actinomyces & Dental caries [34] \\
& Bacillus & Dental caries [35] \\
& Corynebacterium & Biofilm formation [36] \\
& Lactobacillus & Dental caries [34] \\
Micrococcus & Lysozyme activity [37] \\
Mycobacterium & Dental infections [38] \\
Rothia & Dental caries [39] \\
Staphylococcus & Acute sialadenitis [40] \\
Streptococcus & Dental caries [34]
\end{tabular}

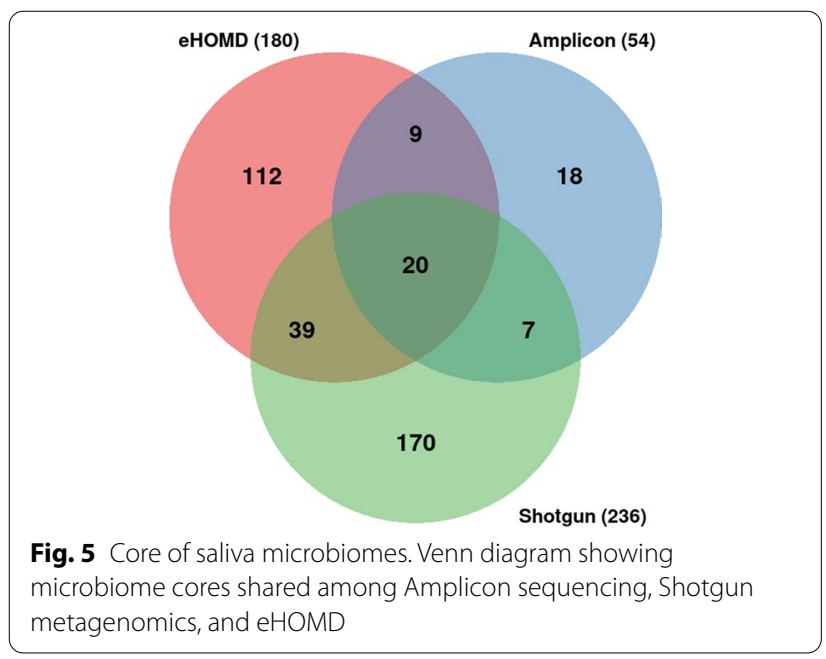

These behaviors in different approaches certainly influenced the comparative study between them (Fig. 1). Furthermore, it was observed in the Amplicon sequencing dataset that all projects did not present sequences with quality failures $($ Failed $=0$ ). This result was unexpected. Even Amplicon sequencing can have quality failures in the sequencing process. However, the Predicted was the only one that did not show difference between the approaches, showing results similar to those observed by
[47], who investigated the microbial composition of the human intestine.

Results obtained by Shotgun metagenomics allowed a more complex characterization of the microbiome, with the identification of greater diversity and at the taxonomic level of species, when compared to Amplicon sequencing which uses regions of the gene with variability to identify down to the genus level [48].

According to the literature [47, 49], the PCA identified a greater number of representative genera in the Shotgun dataset than in the Amplicon dataset (Fig. 3). The differences between them may explain the findings. In Shotgun metagenomics, the DNA of all the organisms in the sample is extracted and sequenced directly. On the other hand, in Amplicon sequencing, only the DNA fragments that were aligned to the primer will be sequenced. The choice of primer seems to be a crucial factor to avoid bias in taxonomic analysis [50].

The specificity of primers may restrict the set of microorganisms found in studies of Amplicon sequencing. Thus, the choice of the sequencing method as well as the selection of primers are important characteristics to be considered in the analysis of microbiome studies [51].

Microbiome studies comparing the two sequencing methods for the same samples suggest that their results might be comparable. In this study, we observed that the data produced by Shotgun metagenomics of salivary samples available on the MG-RAST platform can provide the identification of a greater number of genera, evidencing the complexity of the oral microbiome, either by the diversity of genera or by the role they may play in the salivary microbiome [50].

These results should be interpreted with caution, since only the presence of the genera does not determine the condition of the host. Other characteristics such as abundance and interaction between genera have a relevant role in the association of the microbiota with the condition of the host [12].

Metagenomics projects deposited in public databases such as eHOMD and MG-RAST do not always provide information on the health conditions of the host, DNA/ RNA extraction techniques or other information that might infer microbiome-host relationships.

Studies identified using the information of the principal investigator suggest that they correspond to the data obtained in the MG-RAST. However, it is not possible to specify whether such articles refer to data investigated in this study. According to the MG-RAST pipeline guideline (https://help.mg-rast.org/user_manual.html), it is not possible to carry out analyses of eukaryotes or viruses, which suggests that the DNA/RNA extraction method of the selected projects allows inferring the bacterial microbiota. 


\section{Conclusions}

This study demonstrated that in the microbiota representative of human saliva, genera of pathogenic bacteria observed in oral diseases were identified, but not limited to them.

Core of the salivary microbiome and genera diversity are dependent on the sequencing approaches. Available data suggest that Shotgun metagenomics and Amplicon sequencing have similar sensitivities to detect the taxonomic level investigated, although Shotgun metagenomics allows a deeper analysis of the microorganism diversity.

The choice of metagenomics approaches must consider their characteristics and limitations. Shotgun metagenomics sequencing can provide a great contribution to the knowledge of the composition of the salivary microbiota, identification of markers for diagnosis and identification of profiles capable of defining health or disease conditions. On the other hand, Amplicon sequencing can be an efficient and low-cost choice in studies in which the microorganism of interest is already known. It can also be used for further verification of results obtained by Shotgun metagenomics.

\section{Abbreviations}

NGS: New generation sequencing.; PCR: Polymerase chain reaction.; PCA: Principal components analysis.; eHOMD: Expanded Human Oral Microbiome Database.

\section{Supplementary Information}

The online version contains supplementary material available at https://doi. org/10.1186/s12903-021-01719-5.

Additional file 1: S1 - Microbiome core: Shotgun and Amplicon. Text file with a list of genera belongs to the microbiome core of Shotgun metagenomics and Amplicon sequencing.

Additional file 2: S2 - Microbiome core among Shotgun, Amplicon, and eHOMD. Text file with a list of genera belongs to the microbiome core among Shotgun metagenomics, Amplicon sequencing, and eHOMD.

\section{Acknowledgements}

Rio de Janeiro State University, Piracicaba Dental School, State University of Campinas and Oswaldo Cruz Foundation.

\section{Authors' contributions \\ SGO:planning study, experimental design, experimental work, litera- ture research, data analysis, manuscript preparation, manuscript edit- ing. RRN:experimental work, data analysis. CACT:experimental work. ALMG:manuscript preparation, manuscript revision. AMRD:manuscript preparation, manuscript revision. RJ:planning study, experimental design, experimental work, literature research, data analysis, manuscript preparation, manuscript editing. FHBA: manuscript preparation, manuscript revision. All authors read and approved the final manuscript.}

\section{Funding}

Not applicable.

\section{Availability of data and materials}

Data was deposited in the Mendeley Data (https://doi.org/10.17632/ vvwmvvxxs4.1). Scripts are available at https://github.com/rodrigojardim/ mgrast-search.

\section{Declarations}

\section{Ethics approval and consent to participate}

Not applicable.

\section{Consent for publication \\ Not applicable.}

\section{Competing interest}

The authors declare that they have no competing interests.

\section{Author details}

${ }^{1}$ Department of Restorative Dentistry, Piracicaba Dental School, State University of Campinas, Av. Limeira, 901, Piracicaba, Brazil. ${ }^{2}$ Faculty of Dentistry, Rio de Janeiro State University, Boulevard 28 de setembro, 157, Rio de Janeiro, Brazil. ${ }^{3}$ Laboratory of Diphtheria and Corynebacteria of Clinical Relevance, Faculty of Medical Sciences, Rio de Janeiro State University, Boulevard 28 de setembro, 77, Rio de Janeiro, Brazil. ${ }^{4}$ Computational and Systems Biology Laboratory, Oswaldo Cruz Institute, Oswaldo Cruz Foundation, Av. Brasil, 4365, Rio de Janeiro, Brazil.

Received: 29 January 2021 Accepted: 7 July 2021

Published online: 16 July 2021

\section{References}

1. Handelsman J, Rondon MR, Brady SF, Clardy J, Goodman RM. Molecular biological access to the chemistry of unknown soil microbes: a new frontier for natural products. Chem Biol. 1998:5:R245-9.

2. Tierney BT, et al. The landscape of genetic content in the gut and oral human microbiome. Cell Host Microbe. 2019:26:283-295.e8.

3. Fróes AM, da Mota FF, Cuadrat RRC, Dávila AMR. Distribution and classification of serine $\beta$-lactamases in Brazilian hospital sewage and other environmental metagenomes deposited in public databases. Front Microbiol. 2016;7:1790.

4. Mardis ER. Next-generation DNA sequencing methods. Annu Rev Genom Hum Genet. 2008;9:387-402.

5. Leinonen $R$, Sugawara $H$, Shumway $M$. The sequence read archive. Nucl Acids Res. 2011;39:D19-21.

6. Meyer F, et al. The metagenomics RAST server-a public resource for the automatic phylogenetic and functional analysis of metagenomes. BMC Bioinform. 2008:9:386.

7. Handsley-Davis M, Jamieson L, Kapellas K, Hedges J, Weyrich LS. The role of the oral microbiota in chronic non-communicable disease and its relevance to the Indigenous health gap in Australia. BMC Oral Health. 2020;20:327.

8. Zhang $X$, et al. The oral and gut microbiomes are perturbed in rheumatoid arthritis and partly normalized after treatment. Nat Med. 2015;21:895-905.

9. Fini MB. Oral saliva and COVID-19. Oral Oncol. 2020;108:104821.

10. Streckfus CF. Advances in salivary diagnostics. Advances in salivary diagnostics. Berlin: . Springer; 2015. https://doi.org/10.1007/ 978-3-662-45399-5.

11. Suárez Moya A. Microbioma y secuenciación masiva. Span J Chemother. 2017;30:305-11.

12. Dewhirst FE, et al. The human oral microbiome. J Bacteriol. 2010;192:5002-17.

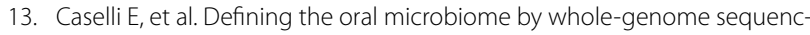
ing and resistome analysis: the complexity of the healthy picture. BMC Microbiol. 2020. https://doi.org/10.1186/s12866-020-01801-y.

14. Wang S, Yan Z, Wang P, Zheng X, Fan J. Comparative metagenomics reveals the microbial diversity and metabolic potentials in the sediments and surrounding seawaters of Qinhuangdao mariculture area. PLoS ONE. 2020;15:e0234128. 
15. Nibali $L$, et al. Differences in the periodontal microbiome of successfully treated and persistent aggressive periodontitis. J Clin Periodontol. 2020;47:980-90

16. Nomura Y, Otsuka R, Hasegawa R, Hanada N. Oral microbiome of children living in an isolated area in Myanmar. Int J Environ Res Public Health. 2020;17:4033-44.

17. Papapanou PN, et al. Subgingival microbiome and clinical periodontal status in an elderly cohort: the WHICAP ancillary study of oral health. J Periodontol. 2020;91:S56-67.

18. Tanner ACR, Kressirer CA, Rothmiller S, Johansson I, Chalmers NI. The caries microbiome: implications for reversing dysbiosis. Rev Adv Dent Res. 2018;29:78-85.

19. Shade A, Handelsman J. Beyond the venn diagram: the hunt for a core microbiome. Environ Microbiol. 2012;14:4-12.

20. Ugland KI, Gray JS. Lognormal distributions and the concept of community equilibrium. Oikos. 1982;39:171-8.

21. Ranjan R, Rani A, Metwally A, McGee HS, Perkins DL. Analysis of the microbiome: advantages of whole genome shotgun versus $16 \mathrm{~S}$ amplicon sequencing. Biochem Biophys Res Commun. 2016;469:967-77.

22. Escapa IF, et al. New insights into human nostril microbiome from the expanded human oral microbiome database (eHOMD): a resource for the microbiome of the human aerodigestive tract. mSystems. 2018;3:e00187-18.

23. $R$ Core Team. $R$ : a language and environment for statistical computing. ( $R$ Foundation for Statistical Computing, 2020).

24. Zhang W, et al. Salivary microbial dysbiosis is associated with systemic inflammatory markers and predicted oral metabolites in non-small cell lung cancer patients. J Cancer. 2019;10:1651-62.

25. Dafar A, Bankvall M, Cevik-Aras H, Jontell M, Sjöberg F. Lingual microbiota profiles of patients with geographic tongue. J Oral Microbiol. 2017:9:1355206

26. Sharma D, et al. Saliva microbiome in primary Sjögren's syndrome reveals distinct set of disease-associated microbes. Oral Dis. 2020;26:295-301.

27. Pereira CA, et al. Opportunistic microorganisms in individuals with lesions of denture stomatitis. Diagn Microbiol Infect Dis. 2013;76:419-24

28. Guerrero-Preston $\mathrm{R}$, et al. $16 \mathrm{~S}$ rRNA amplicon sequencing identifies microbiota associated with oral cancer, Human Papilloma Virus infection and surgical treatment. Oncotarget. 2016;7:51320-34.

29. de Melo F, Milanesi FC, Angst PDM, Oppermann RV. A systematic review of the microbiota composition in various peri-implant conditions: data from 16S rRNA gene sequencing. Arch Oral Biol. 2020;117:104776.

30. Yamashita Y Takeshita T. The oral microbiome and human health. J Oral Sci. 2017;59:201-6.

31. Kakabadze MZ, Paresishvili T, Karalashvili L, Chakhunashvili D, Kakabadze Z. Oral microbiota and oral cancer: review. Oncol Rev. 2020;14:476.

32. Sun $X$, et al. Alteration of salivary microbiome in periodontitis with or without type-2 diabetes mellitus and metformin treatment. Sci Rep. 2020;10:1-14.

33. Yu FY, et al. Dysbiosis of saliva microbiome in patients with oral lichen planus. BMC Microbiol. 2020;20:75.
34. Gao X, Jiang S, Koh D, Hsu CYS. Salivary biomarkers for dental caries. Periodontol. 2016;2000(70):128-41.

35. Ratna Sudha M, Neelamraju J, Surendra Reddy M, Kumar M. Evaluation of the effect of probiotic bacillus coagulans unique IS2 on mutans streptococci and lactobacilli levels in saliva and plaque: a doubleblind, randomized, placebo-controlled study in children. Int J Dent. 2020;2020:8891708.

36. Esberg A, et al. Corynebacterium matruchotii demography and adhesion determinants in the oral cavity of healthy individuals. Microorganisms. 2020;8:1780.

37. Gennari CGM, Sperandeo P, Polissi A, Minghetti P, Cilurzo F. Lysozyme mucoadhesive tablets obtained by freeze-drying. J Pharm Sci. 2019;108:3667-74.

38. Cortela DCB, Souza Junior ALD, Virmond MCL, Ignotti E. Inflammatory mediators of leprosy reactional episodes and dental infections: a systematic review. Mediators Inflamm. 2015;1-15.

39. Vieira $A R$, et al. Profiling microorganisms in whole saliva of children with and without dental caries. Clin Exp Dent Res. 2019:5:438-46.

40. Ogle OE. Salivary gland diseases. Dent Clin North Am. 2020;64:87-104.

41. Zhang Y, et al. Human oral microbiota and its modulation for oral health. Biomed Pharmacother. 2018;99:883-93.

42. Belstrøm D. The salivary microbiota in health and disease. J Oral Microbiol. 2020;12:1-7.

43. Belstrøm D, et al. Salivary microbiota in individuals with different levels of caries experience. J Oral Microbiol. 2017:9:1-8.

44. Willmann C, et al. Oral health status in historic population: Macroscopic and metagenomic evidence. PLOS ONE. 2018;13:1-18.

45. Tran $T L Q$, et al. Chitinophaga vietnamensis sp. Nov., a multi-drug resistant bacterium infecting humans. Int J Syst Evol Microbiol. 2020;70:1758-68.

46. Crémet $L$, et al. Chitinophaga terrae bacteremia in human. Emerg Infect Dis. 2009;15:1134-5.

47. Laudadio I, et al. Quantitative assessment of shotgun metagenomics and 165 rDNA Amplicon sequencing in the study of human gut microbiome. OMICS J Integr Biol. 2018;22:248-54.

48. Wade WG. The oral microbiome in health and disease. Pharmacol Res. 2013:69:137-43.

49. Jovel J, et al. Characterization of the gut microbiome using 165 or shotgun metagenomics. Front Microbiol Wwwfrontiersinorg. 2016;1:459-76.

50. Rintala A, et al. Gut microbiota analysis results are highly dependent on the 16s rRNA gene target region, whereas the impact of DNA extraction is minor. J Biomol Tech. 2017;28:19-30.

51. Clarridge JE. Impact of $16 \mathrm{~S}$ rRNA gene sequence analysis for identification of bacteria on clinical microbiology and infectious diseases. Clin Microbiol Rev. 2004;17:840-62.

\section{Publisher's Note}

Springer Nature remains neutral with regard to jurisdictional claims in published maps and institutional affiliations.
Ready to submit your research? Choose BMC and benefit from:

- fast, convenient online submission

- thorough peer review by experienced researchers in your field

- rapid publication on acceptance

- support for research data, including large and complex data types

- gold Open Access which fosters wider collaboration and increased citations

- maximum visibility for your research: over $100 \mathrm{M}$ website views per year

At $\mathrm{BMC}$, research is always in progress.

Learn more biomedcentral.com/submissions 